\title{
Concerns about health care warnings and their impact on prescribing behaviour
}

\author{
Laurence Y. Katz MD
}

$\infty \quad$ See related research paper by Valiyeva and colleagues, page 438

I $\mathrm{n}$ this issue of $C M A J$ Valiyeva and colleagues ${ }^{1}$ report on the impact of 3 regulatory warnings on prescription rates of antipsychotic drugs among elderly adults with dementia. Their finding — that the warnings did not achieve the desired outcome - is similar to that in a recent study on the use of antidepressants in children and adolescents. ${ }^{2}$ The similarity illustrates a lack of effectiveness of health care warnings in achieving the desired outcome through communicating information on morbidity and mortality risks in addition to concerns about efficacy, especially in our most vulnerable patient populations - youths and elderly people..$^{2-4}$ This problem has multiple contributors, and its resolution will likely require cooperation among regulatory agencies, the pharmaceutical industry, professional organizations representing physicians and, ultimately, physicians themselves.

For decades, conventional and more recently atypical antipsychotic drugs have been used, primarily "off-label," to reduce the behavioural problems experienced by elderly patients with dementia. ${ }^{4}$ Currently, only risperidone has been approved by Health Canada for this indication; none has been approved for such use by the US Food and Drug Administration. A well-designed randomized controlled trial and a recent consensus conference have suggested that the efficacy of conventional and atypical antipsychotic drugs for this indication has not been shown. ${ }^{4,5}$ To identify the lack of efficacy, regulatory agencies and authors had to get access to unpublished reports of trials, most of which were the property of the pharmaceutical companies that had conducted the studies. ${ }^{6}$ Furthermore, a meta-analysis of all published and unpublished studies as well as large, well-designed ecological studies have indicated an increased risk of death among elderly patients with dementia taking antipsychotic drugs. ${ }^{3,6,7}$ These findings prompted the mailing of 3 warnings to Canadian physicians about the risks associated with the use of atypical antipsychotic agents in elderly patients with dementia. This process is almost identical to one that unfolded regarding the use of antidepressants among children and adolescents with depression. ${ }^{2}$ In that study, concerns were raised that the warnings resulted in decreased provision of care even though they had the desired effect of decreased rates of use of antidepressants in these age groups. ${ }^{2}$

In the study by Valiyeva and colleagues, ${ }^{1}$ the authors used comprehensive population-based administrative health care databases and a longitudinal study design to evaluate the prescription rates of conventional and atypical antipsychotic drugs among elderly patients with dementia before and after each of the 3 warnings. They found that use of atypical antipsychotic drugs was on the rise before the first warning. After each warning, they found a slight slowing in the rate of

\section{Key points}

- Conventional and atypical antipsychotic drugs are used ex tensively to treat behavioural disturbances in elderly patients with dementia.

- The efficacy of antipsychotic drugs for this purpose has not been shown, and the treatment is associated with an increased risk of death in this patient population.

- Health care warnings about the risks have not significantly altered prescription rates of atypical antipsychotic drugs among elderly patients with dementia in Ontario.

- Health care warnings should provide complete information about the risks and efficacy of the identified treatment and of alternative treatments.

- Mechanisms to evaluate the impact of regulatory warnings and whether they achieve their desired effect are needed.

growth in their use; however, the overall prescription rate of these drugs continued to increase over the study period, which indicated that the warnings had a minimal impact on prescribing behaviour. In the study of antidepressant use among children and adolescents with depression, the prescribing rate among adolescents levelled off over the 2 years after the regulatory warning was issued, but it was still twice the rate observed 9 years before the warning. ${ }^{2}$

Thus, we have a scenario in which physicians and their patients are unaware of all the data on efficacy (or lack thereof); concerns have been raised about incomplete reporting of adverse events in randomized controlled trials by a pharmaceutical company ${ }^{8}$ and postmarketing information on morbidity and mortality risks are not effectively communicated to physicians in a manner that will lead to the desired outcomes. ${ }^{1,2}$ Furthermore, no mechanisms are in place to evaluate the impact of health care warnings and whether they achieve the desired effects.

This scenario is not unique to mental health; it also exists in other areas of medicine..$^{9,10}$ In fact, it could present a threat to the very premise of evidence-based medicine. Unfortunately, because there are no mechanisms in place to evaluate the effectiveness of health care warnings, there is a paucity of data on their impact, so the extent of the problem is unknown.

Why do some health care warnings fail to achieve the desired results? The warnings about the use of antidepressants in children and adolescents and of atypical antipsychotic medications in elderly patients with dementia delineated concerns about the associated morbidity and mortality risks. However, the warnings did not provide information about the

Laurence Katz is an associate professor in the Department of Psychiatry، University of Manitoba, Winnipeg, Man. 
effectiveness and safety of alternative treatment options. This lack of information is not helpful to physicians when confronted with patients, families and allied health care professionals in understandable distress regarding the patient's symptoms. The results of the study by Valiyeva and colleagues ${ }^{1}$ suggest that physicians will return to what they know, despite documented risks, if they are not given other options. Furthermore, in a study comparing the effect of wording of different health care warnings on the concomitant prescribing of cisapride with contraindicated medications, Weatherby and colleagues ${ }^{11}$ suggested that the key characteristics of a successful warning appear to be explicit identification of the medications and their risks, and publicity of the warning. However, although the warnings about antidepressant use in children and adolescents had both of these key characteristics, they had unintended adverse consequences on the provision of care and clinical outcomes. This suggests that explicit identification and publicity are not sufficient to achieve the desired effects and avoid unintended outcomes.

So what do we do? Clearly, regulatory agencies must monitor the ongoing safety of approved treatments and, when appropriate, issue health care warnings as a necessary component of a safe health care system. However, warnings must communicate more than just the risks associated with a particular treatment. Decisions about treatment always involve balancing risks and benefits. Thus, health care warnings should provide accurate, explicit and complete information about the risks and efficacy of the identified treatment in addition to providing the same information on alternative treatments. ${ }^{12}$ For example, in patients with dementia, there are alternative pharmacologic and behavioural treatments that could be considered (although these options also have limited evidence to support their use). Furthermore, whatever the specific nature of health care warnings, a mechanism to evaluate their impact and whether they achieve the desired effect is needed. There must then be a mechanism to remedy situations in which the desired effect has not occurred.

To achieve such a system, Health Canada will likely have to collaborate with the pharmaceutical industry and professional organizations that represent physicians. Health Canada will require access to all data (published and unpublished) from the pharmaceutical industry and physicians, as well as their assistance to develop effective mechanisms of communicating the risks and benefits of identified treatments. This collaboration could also assist with the development of mechanisms to evaluate the impact of the warnings and remedies for any unforeseen problems.

Competing interests: None declared.

\section{REFERENCES}

1. Valiyeva E, Herrmann N, Rochon PA, et al. Effect of regulatory warnings on antipsychotic prescription rates among elderly patients with dementia: a populationbased time-series analysis. CMAJ 2008;179:438-46.

2. Katz LY, Kozyrskyj AL, Prior HJ, et al. Effect of regulatory warnings on antidepressant prescription rates, use of health services and outcomes among children, adolescents and young adults. CMAJ 2008;178:1005-11.

3. Gill SS, Bronskill SE, Normand SLT, et al. Antipsychotic drug use and mortality in older adults with dementia. Ann Intern Med 2007;146:775-86.

4. Salzman C, Jeste DV, Meyer RE, et al. Elderly patients with dementia-related symptoms of severe agitation and aggression: consensus statement on treatment options, clinical trials methodology and policy. J Clin Psychiatry 2008;May 13:e1e10. [Epub ahead of print]

5. Schneider LS, Tariot PN, Davis SM, et al. Effectiveness of atypical antipsychotic drugs in patients with Alzheimer's disease. N Engl J Med 2006;355:1525-38.

6. Schneider LS, Dagerman KS, Insel P. Risk of death with atypical antipsychotic drug treatment for dementia, meta-analysis of randomized placebo-controlled trials. JAMA 2005;294:1934-43.

7. Wang PS, Schneeweiss S, Avorn J, et al. Risk of death in elderly users of conventional vs. atypical antipsychotic medications. N Engl J Med 2005;353:2335-41.

8. US Food and Drug Administration. Re: NDA \# 20-639, Seroquel (quetiapine fumarate) tablets, MACMIS ID \# 14670 [FDA warning letter]. Rockville (MD): The Administration; 2006. Available: www.fda.gov/cder/warn/2006/Seroquel_letter.pdf (accessed 2008 June 24).

9. Smalley W, Shatin D, Wysowski DK, et al. Contraindicated use of cisapride: impact of food and drug administration regulatory action. JAMA 2000;284:3036-9.

10. Hauptman PJ, Schnitzler MA, Swindle J, et al. Use of nesiritide before and after publications suggesting drug-related risks in patients with acute decompensated heart failure. JAMA 2006;296:1877-84.

11. Weatherby LB, Nordstrom BL, Fife D, et al. The impact of wording in "Dear Doctor" letters and in black box labels. Clin Pharmacol Ther 2002;72:735-42.

12. Mamdani MM. Health advisories: when good intentions go bad [editorial]. CMAJ 2008;178:1025-6.

Correspondence to: Dr. Laurence Y. Katz, Department of Psychiatry, University of Manitoba, PZ-162, 771 Bannatyne Ave., Winnipeg MB R3E 3N4; fax 204 787-4975; lkatz@hsc.mb.ca 